\title{
Visual and Chemical Prey Cues as Complementary Predator Attractants in a Tropical Stream Fish Assemblage
}

\author{
Chris K. Elvidge and Grant E. Brown \\ Department of Biology, Concordia University, 7141 Sherbrooke St. West, Montreal, QC, Canada H4B 1R6 \\ Correspondence should be addressed to Chris K. Elvidge, chris.k.elvidge@gmail.com
}

Received 27 March 2012; Revised 25 April 2012; Accepted 30 April 2012

Academic Editor: Marie Herberstein

Copyright ( $) 2012$ C. K. Elvidge and G. E. Brown. This is an open access article distributed under the Creative Commons Attribution License, which permits unrestricted use, distribution, and reproduction in any medium, provided the original work is properly cited.

\begin{abstract}
To date, little attention has been devoted to possible complementary effects of multiple forms of public information similar information on the foraging behaviour of predators. In order to examine how predators may incorporate multiple information sources, we conducted a series of predator attraction trials in the Lower Aripo River, Trinidad. Four combinations of visual (present or absent) and chemical cues (present or absent) from each of two prey species were presented. The occurrences of three locally abundant predatory species present within a $1 \mathrm{~m}$ radius of cue introduction sites were recorded. The relative attractiveness of cue type to each predator was directly related to their primary foraging modes, with visual ambush predators demonstrating an attraction to visual cues, benthivores to chemical cues, and active social foragers demonstrating complementary responses to paired cues. Predator species-pair counts were greatest in response to cues from the more abundant prey species, indicating that individuals may adopt riskier foraging strategies when presented with more familiar prey cues. These differences in predator attraction patterns demonstrate complementary effects of multiple sensory cues on the short-term habitat use and foraging behaviour of predators under fully natural conditions.
\end{abstract}

\section{Introduction}

The behavioural strategies adopted by participants in predator-prey interactions are often mediated by publicly available cues [1] conveying information with some degree of immediate contextual relevance to the receiver. Public, or non-species-specific, cues may convey qualitatively different information to and elicit quantitatively different behavioural responses from different receivers [2]. The relative importance of different types of public cues in predator-prey interactions may be mediated by interactions between receiver taxon and environmental constraints; for example, visual cues are typically limited by photoperiod [3]. In aquatic environments, visual and chemical cues have been identified as the primary sources of information eliciting short-term behavioural processes for both vertebrate (e.g., fishes, [4]) and invertebrate (e.g., crustaceans, $[5,6]$ ) species. Although acoustic cues have been demonstrated to elicit behavioural responses in freshwater fish receivers under laboratory conditions [7], the reliability of acoustic information may be limited under conditions of relatively high background noise, as in lotic systems.

Many groups of freshwater fishes produce chemical cues in the epidermis which are released into the water following mechanical damage, as would occur during a predation event [8]. Upon detection by conspecific receivers, these cues have been shown to elicit a suite of antipredator or alarm responses [9] in centrarchid [10], salmonid [11], cyprinid [12], cyprinodontiform [2], esocid [13], and poeciliid [14] species. Due to their manner of release, these chemical cues cannot be manipulated by a predator and likely serve as reliable indicators of increased risk to receivers subject to similar predation pressures [15], which is not always the case with potentially misleading visual cues [3]. Damagereleased chemical cues have been shown to elicit different responses from conspecific receivers differing in ontogenetic stage from the cue sender [16], with similarly sized receivers demonstrating alarm or antipredator responses and larger receivers demonstrating behaviours consistent with foraging responses under laboratory conditions. Similar effects have 
been observed in heterospecific receivers belonging to the same prey guild and subject to similar predation risks as chemical cue senders $[17,18]$. Conversely, heterospecific receivers of larger size than the sender have demonstrated foraging responses following exposure to damage-released chemical cues under both laboratory [18] and field [19] conditions.

Due to the potentially ultimate costs incurred by failing to respond to ambient cues indicating elevated predation risk, the responses of prey to public cues have thus far received considerably more attention from researchers than have responses by predators [20]. In freshwater fishes, laboratory experiments have documented attraction responses to chemical cues from heterospecific prey [21], while predators under natural conditions have demonstrated preferences for areas labelled with damage-released chemical cues over longer time scales (hours, [19]). Recently, Lonnstedt et al. [22] demonstrated an attraction response in a predatory coral reef fish, the dottyback Pseudochromis fuscus to heterospecific damage-released chemical cues under fully natural conditions over short (minutes) timescales. In addition, under both natural and laboratory conditions, $P$. fuscus demonstrated a strong preference for chemical cues extracted from heterospecific donors belonging to the ideal prey size class [22] for gape-limited predators [17]. Similarly, Elvidge et al. [2] demonstrated significant positive linear relationships between foraging behaviours and receiver size in an opportunistic predator, Hart's rivulus Rivulus hartii, in response to chemical cues from Trinidadian guppies Poecilia reticulata. Together, these results indicate that damagereleased chemical cues provide predators with information about the availability and quality of potential prey.

In order to examine the effects of different combinations of visual and chemical cues indicating the availability of prey on the behaviour of predators in fresh water, the present study focused on short-term changes in local abundances of three predatory species differing in foraging modes to the cues of two cooccurring prey species. In general, we predict that multiple complementary cues indicating the presence of familiar prey species will result in greater local abundances of predators, with the relative contribution of each type of cue (visual or chemical) mediated by the typical foraging mode of the predator.

\section{Materials and Methods}

2.1. Study Species and Area. Predator attraction trials were conducted at $N=16$ sites in a series of eight pools (two sites per pool) along a $1 \mathrm{~km}$ stretch of the Lower Aripo River in the Caroni drainage, Northern Range Mountains, Trinidad and Tobago, W. I. (10³9' N, 61 ${ }^{\circ} 13^{\prime}$ W) 04-12 May 2009. These pools have been described in an earlier study involving free-swimming Trinidadian guppies $P$. reticulata [23]. The Lower Aripo is a species-rich, high-predation environment [24] with abundant $P$. reticulata and incidental $R$. hartii populations. These two prey species are nearly ubiquitous in streams in northern Trinidad but do not always cooccur [25]. Although $R$. hartii may grow to as much as three times the length of $P$. reticulata ( $R$. hartii maximum standard length, $L_{S}, 100 \mathrm{~mm}$, $P$. reticulata common $L_{S} 28 \mathrm{~mm}$; [26]) and $P$. reticulata can account for up to $10 \%$ of the diet of large $R$. hartii, in the presence of piscivores similarly sized $P$. reticulata and $R$. hartii are likely subject to similar predation pressures [17].

Of the three predatory species examined, the pike cichlid Crenicichla alta is a solitary, visually foraging ambush predator and obligate piscivore which is considered the main predator of $P$. reticulata in Trinidad whenever they co-occur [25]. The blue acara cichlid Aequidens pulcher is a solitary forager which typically feeds on invertebrates and benthos, displaying only opportunistic piscivory [27]. The two-spot sardine Astyanax bimaculatus, by contrast, is a highly social and active forager $[23,26]$, whose predominantly algaeand insect-based diet undergoes an ontogenetic switch to include opportunistic piscivory when individuals exceed $\sim 50 \mathrm{~mm}$ total length [28]. These three species likely account for the majority of predation pressure on $P$. reticulata and any incidental $R$. hartii within the study area [29]. Based on these differences in social behaviour and foraging mode, we predict that $A$. bimaculatus incorporates information received through both visual and chemical cues into their foraging decisions, while $A$. pulcher responds more strongly to chemical than to visual cues and C. alta responds primarily to visual cues.

2.2. Prey Cues. Damage-released chemical cues were extracted from female $P$. reticulata $\left(L_{S} 27.6 \pm 2.7 \mathrm{~mm}\right.$ (mean \pm $\mathrm{SD}), N=18$ ) and $R$. hartii $\left(L_{S} 42 \pm 7.9 \mathrm{~mm}\right.$ (mean $\left.\pm \mathrm{SD}\right)$, $N=8$ ) donors collected from the Naranja River tributary $\left(10^{\circ} 41^{\prime} \mathrm{N} ; 61^{\circ} 14^{\prime} \mathrm{W}\right)$ approximately $6 \mathrm{~km}$ upstream from the observation sites in the Lower Aripo River using a beach seine net (length $2.5 \mathrm{~m}$, height $1 \mathrm{~m}$, mesh size $3 \mathrm{~mm}$ ). Donors were collected from the Naranja River because intensive sampling via seine net did not find any $R$. hartii present at the study sites in the Lower Aripo during the course of the present experiment. Chemical cues from $P$. reticulata donors from the Naranja River have previously been demonstrated to elicit qualitatively similar responses to those of Lower Aripo donors [23] in conspecific receivers from either population.

Chemical cue donors were euthanized via cervical dislocation, measured $\left(L_{S}\right)$, immediately decapitated behind the opercula, and had their tails removed at the caudal peduncle. Visceral tissues were manually extruded, and the remaining carcasses were mechanically homogenized in dechlorinated tap water, diluted to a final concentration of $0.1 \mathrm{~cm}^{2}$ skin $\mathrm{mL}^{-1}$, and filtered through polyester floss. This concentration of skin extract has previously been shown to elicit both antipredator and foraging behavioural responses in tropical stream fish under laboratory [30] and field [23] conditions. The chemical cues from each prey species, as well as a stream water control treatment, were packaged in $60 \mathrm{~mL}$ aliquots and frozen at $-20^{\circ} \mathrm{C}$ until use.

Several female $P$. reticulata $\left(L_{S} 27.9 \pm 2.9 \mathrm{~mm}\right.$ (mean \pm $\mathrm{SD}), N=8$ ) and juvenile $R$. hartii ( $L_{S} 25.4 \pm 4.8 \mathrm{~mm}$ (mean $\pm \mathrm{SD}$ ), $N=8$ ) were retained from the pools of wild-caught chemical cue donors to serve as visual prey 
TABLE 1: Effects of different combinations of cues from two prey species indicating potential foraging opportunities on the local abundance of three predatory species in a nested ANOVA with observation site nested within prey species*.

\begin{tabular}{|c|c|c|c|c|c|c|c|c|c|c|}
\hline \multirow{2}{*}{ Predator } & \multirow{2}{*}{ Prey cue } & \multicolumn{3}{|c|}{ Treatment effects } & \multicolumn{3}{|c|}{ Nested effects } & \multicolumn{3}{|c|}{ Variance components } \\
\hline & & $F$ & df & $P$ & Nested factor & $F$ & $P$ & Prey cue & Prey species & Site \\
\hline \multirow{2}{*}{ C. alta } & Visual & 19.41 & 2,92 & $<0.0001$ & Prey species & 1.02 & 0.3145 & \multirow{2}{*}{$73.1 \%$} & \multirow{2}{*}{$5.2 \%$} & \multirow{2}{*}{$21.7 \%$} \\
\hline & Chemical & 0.09 & 2,92 & 0.91 & Site & 0.05 & 0.8234 & & & \\
\hline \multirow{2}{*}{ A. pulcher } & Visual & 2.12 & 2,92 & 0.126 & Prey species & 0.92 & 0.3394 & \multirow{2}{*}{$65.4 \%$} & \multirow{2}{*}{$17.4 \%$} & \multirow{2}{*}{$17.2 \%$} \\
\hline & Chemical & 6.36 & 2,92 & 0.0026 & Site & 23.58 & $<0.0001$ & & & \\
\hline \multirow{2}{*}{ A. bimaculatus } & Visual & 6.08 & 2,92 & 0.0033 & Prey species & 0.45 & 0.5036 & \multirow{2}{*}{$52.7 \%$} & \multirow{2}{*}{$47.3 \%$} & \multirow{2}{*}{$0 \%$} \\
\hline & Chemical & 19.21 & 2,92 & $<0.0001$ & Site & 6.71 & 0.0108 & & & \\
\hline
\end{tabular}

${ }^{*}$ The interactions between prey cue types were nonsignificant in all tests so the analyses were limited to main and nested effects only.

cues. Subjects were transported to the observation site and placed singly into clear plastic bottles $(250 \mathrm{~mL})$ that had been perforated to allow water exchange. The bottles were attached to $1 \mathrm{~m}$ lengths of wooden dowelling ( $1 \mathrm{~cm}$ diameter) by transparent fishing wire and held stationary in the water column approximately $5 \mathrm{~cm}$ off the substrate. The bottles were also presented empty to serve as a control treatment to the visual prey cues and provide estimates of ambient predator abundance in the presence of observers. Each site $(N=16)$ was presented with the four combinations of cues for each prey species $(N=8$ trials per site) for a total of $N=128$ observations.

2.3. Experimental Protocol and Analysis. Chemical stimuli consisting of $60 \mathrm{~mL}$ of either $P$. reticulata or $R$. hartii chemical cues (CC) or a stream water control (SW) were delivered by syringe through $2 \mathrm{~m}$ lengths of airline tubing anchored by a rock $(5 \mathrm{~cm}$ diameter) placed at the site of an observation. The bottles containing the visual stimuli or visual control were introduced into the water column directly above the stimulus injection sites. The apparatus was left in place for 1-minute prior to an observation to allow nearby fish to acclimate to its presence. Subsequent observations were conducted in an upstream direction to minimize the likelihood of attracting additional predators from the downstream dispersion of the chemical prey cues.

Following the 1 minute acclimation period, a stopwatch was activated to begin a 5-minute observation, throughout which the chemical stimuli or chemical controls were delivered through the airline tubing at a rate of $10 \mathrm{~mL} \mathrm{~min}-1$ and the numbers of individuals of the three predatory species within a $1 \mathrm{~m}$ radius of the cue presentation site were recorded every 15 seconds. These predator counts were then averaged by species over the 5 -minute observation periods. A similar protocol has previously been used to examine the predator inspection behaviour of free-swimming $P$. reticulata under field conditions [23]. The mean counts for each predatory species were subsequently examined as univariate responses in two-way nested ANOVAs with the chemical and visual prey cues as main effects, and replicated observation site nested within prey species. All analyses were conducted as linear mixed-effects lme models using the nlme statistical package [31] for R (version 2.12.1; [32]). The models were then decomposed to determine the relative influence of model components on the variance in response using the varcomp command in the ape library [33]. Secondarily, the dataset was split into two parts by prey species and analyzed as univariate two-way ANOVAs to enable direct comparisons of the attractiveness of visual and chemical prey cues to each predator species. Additionally, in order to examine the possibility that the presence of the top predator, C. alta, at an observation site may have inhibited the attraction of the other predatory species to the area, predator species-pair counts were square-root transformed and compared using Pearson's correlation analyses.

\section{Results}

3.1. Predator Attraction. In no predator versus prey species combination was there a significant interaction between chemical and visual prey cues on predator species counts $(P>0.05)$, so further analyses examined main effects of prey cue types only. Results of two-way nested ANOVAs on the attraction of each predatory species to the combinations of prey cues with observation site as a factor nested within prey species are presented in Table 1. Despite the likelihood of a high degree of spatial heterogeneity in the distribution of predatory species in the Lower Aripo, observation site as a nested factor accounted for $\leq 21.7 \%$ of the variability in mean predator counts (Table 1). The least spatial variability in predator counts was demonstrated by the highly active A. bimaculatus, with the less motile and solitary A. pulcher and C. alta demonstrating greater heterogeneity in their distributions throughout the study sites.

The response patterns of each predator to the chemical and visual cue combinations appear to be similar for both $P$. reticulata and $R$. hartii cues (Figure 1). Prey species accounts for $5-47 \%$ of the variance in mean predator counts within study areas with $C$. alta demonstrating the least difference in response between prey species and A. bimaculatus demonstrating the greatest difference (Table 1). Overall, there appears to be a nonstatistically significant preference for the $P$. reticulata cues as suggested by the greater mean counts of predators within an observation radius relative to the $R$. hartii cues (Figure 1). Prey cue treatments (main effects) accounted for $52.7 \%$ of the variability in mean counts of $A$. bimaculatus and $73.1 \%$ of the variability in C. alta counts. As with the effect of prey species, the portions of variance in $A$. 


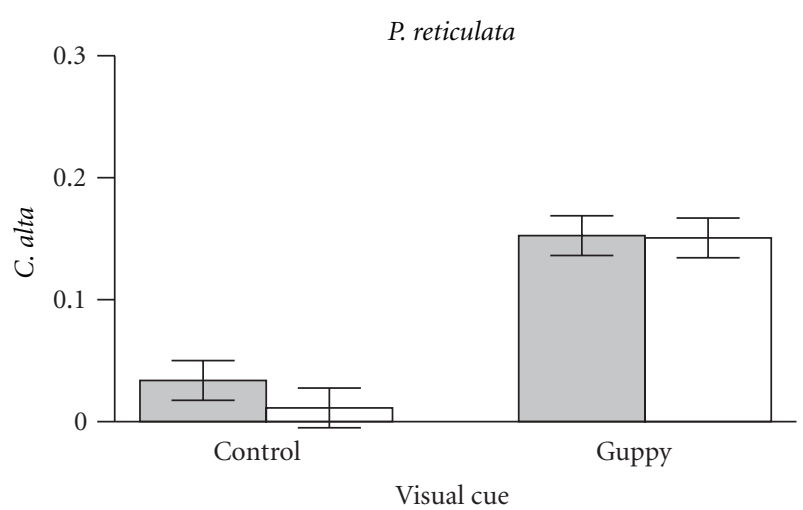

(a)

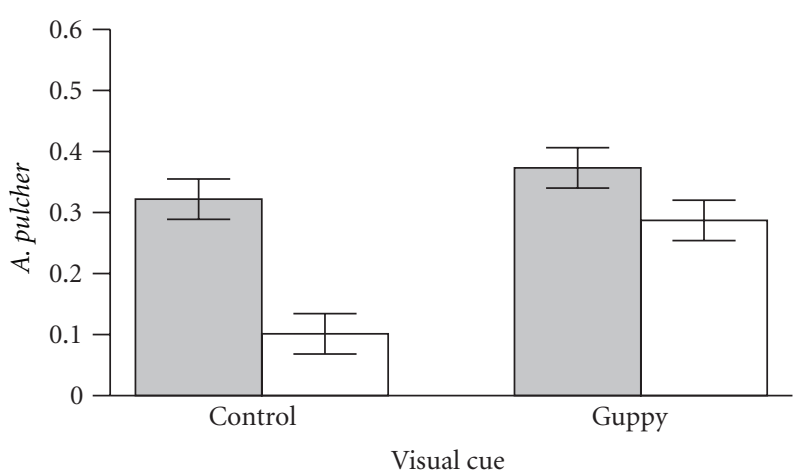

(c)

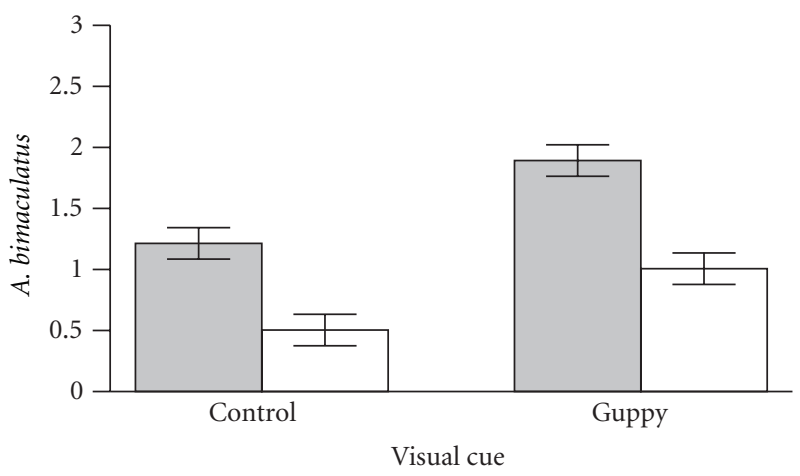

CC

SW

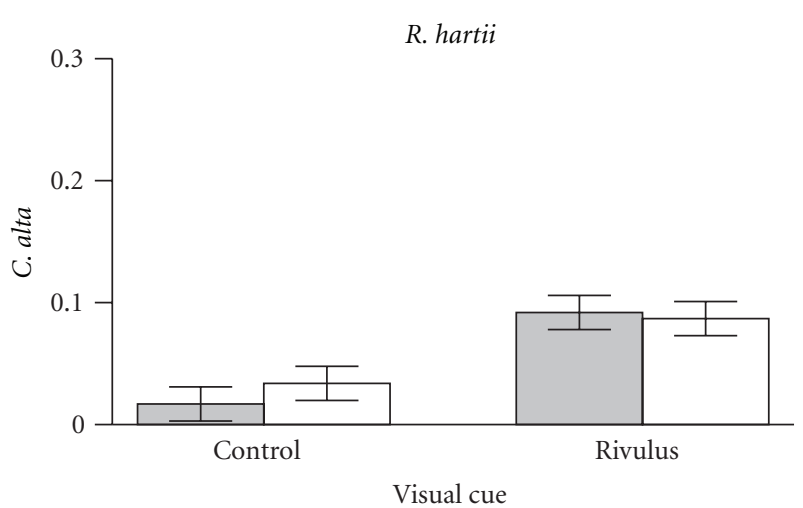

(b)

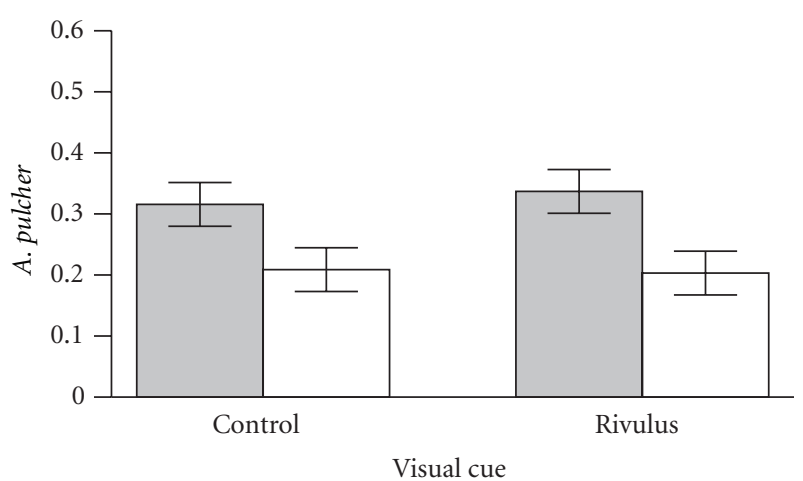

(d)

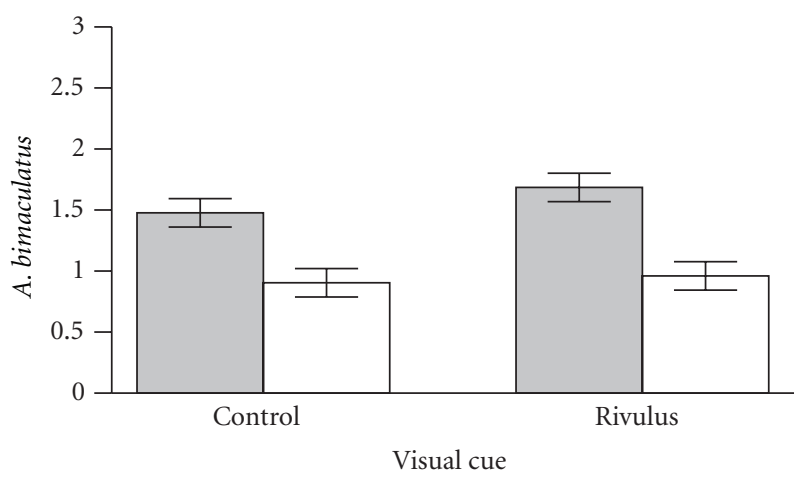

(f)

FIGURE 1: Mean $( \pm$ SE) number of predators present within a $1.5 \mathrm{~m}$ radius of prey cue presentation sites in the Lower Aripo over 5 minutes. Crenicichla alta attraction to (a) guppy Poecilia reticulata and (b) rivulus Rivulus hartii cues. Aequidens pulcher attraction to (c) guppy and (d) rivulus cues. Astyanax bimaculatus attraction to (e) guppy and (f) rivulus cues. Visual cues (horizontal axes) were paired with either conspecific chemical cues (shaded bars) or a stream water control (open bars). $N=16$ for each cue combination.

pulcher responses (65.4\%) were intermediate relative to the other two predators.

As predicted by its primary foraging strategy, Crenicichla alta, a visual ambush predator, was observed in greater numbers when presented with visual cues indicating foraging opportunities of either $P$. reticulata $\left(F_{1,46}=24.3, P<\right.$ 0.0001; Figure 1(a)) or $R$. hartii $\left(F_{1,46}=8.78, P=0.0057\right.$; Figure 1(b)); although its response to $P$. reticulata visual cues appears to be greater than to those of $R$. hartii, the difference is nonsignificant. Crenicichla alta did not demonstrate any attraction to chemical cues from either prey species $(P>$ $0.05)$. This is in keeping with its foraging strategy of ambush hunting, as diffusive chemical cues may not reliably indicate the location of potential prey to visual predators.

Aequidens pulcher responded to both $P$. reticulata $\left(F_{1,46}=\right.$ 6.52, $P=0.014$; Figure $1(\mathrm{c}))$ and $R$. hartii $\left(F_{1,46}=6.25, P=\right.$ 0.016; Figure 1(d)) chemical cues but not to the visual cues of either prey species $(P>0.05)$. A significant response to 


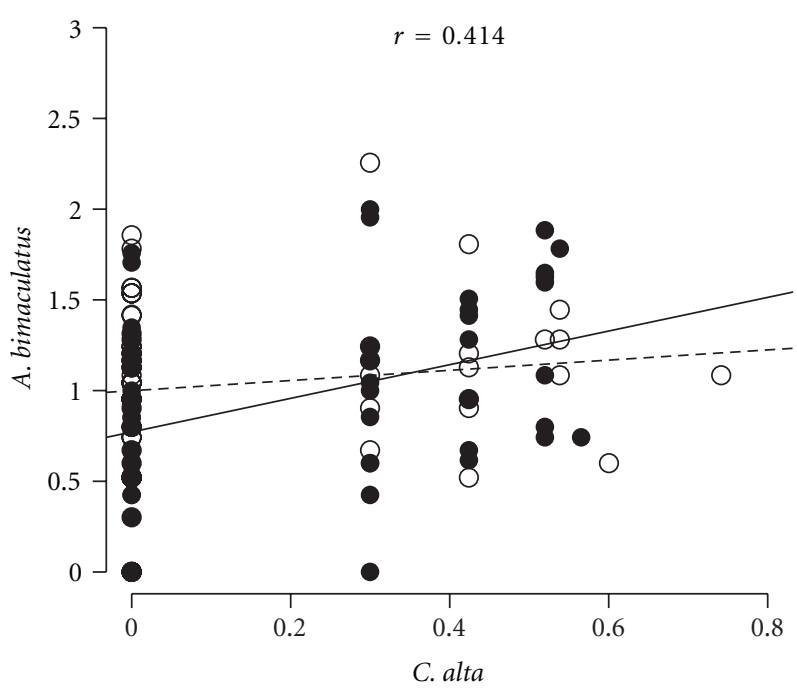

(a)

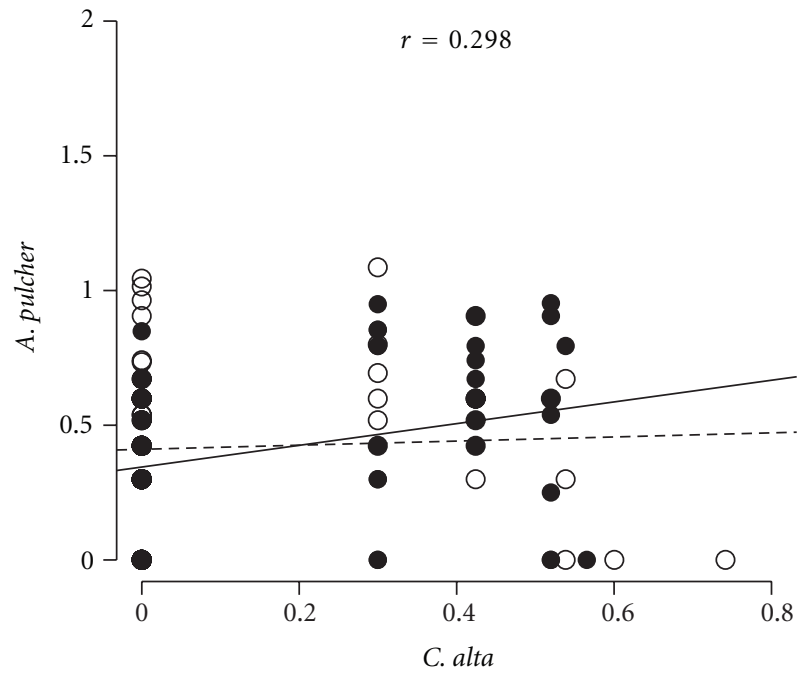

(b)

Figure 2: Pearson's correlation analyses of square-root transformed mean counts of Astyanax bimaculatus (a) and Aequidens pulcher (b) observed within $1 \mathrm{~m}$ radii of the prey cue introduction sites in the presence of the top predator, Crenicichla alta. Poecilia reticulata cues, closed points; Rivulus hartii cues, open points. Significant linear relationships $(P<0.05)$ are indicated by solid lines; nonsignificant relationships are indicated by dashed lines for illustrative purposes.

the chemical but not the visual cues of both prey species is in keeping with the prediction that the importance of chemical cues is greater than visual cues for a bottom-feeding detritivore.

Astyanax bimaculatus demonstrated a response to $P$. reticulata visual cues $\left(F_{1,46}=11.7, P=0.0013\right.$; Figure $\left.1(\mathrm{e})\right)$ but not to $R$. hartii visual cues $(P>0.05)$ and responded to the chemical cues of both prey species ( $P$. reticulata: $F_{1,46}=21.3, P<0.0001 ; R$. hartii: $F_{1,46}=16.9, P=0.0002$; Figure 1(f)). Additionally, the response by A. bimaculatus to complementary chemical and visual cues of both prey species appears to be approximately additive (Figures 1(e) and $1(\mathrm{f})$ ). This social foraging species was alone in this study in demonstrating complementary responses to both types of sensory cues indicating the presence of potential prey.

3.2. Predator Interactions. During trials involving P. reticulata cues, the mean counts of $C$. alta were positively correlated with the counts of both $A$. bimaculatus $(P=$ 0.0007; Figure 2(a)) and A. pulcher $(P=0.017$; Figure 2(b)). Conversely, there were no relationships between the speciespair counts in trials involving the cues of the less locally abundant $R$. hartii (Figure 2).

\section{Discussion}

These results demonstrate the importance of foraging mode in determining the relative influence of different types of prey cues on predator behaviour and habitat use. Introducing chemical and visual prey cues resulted in increased local abundances of predators, with the demonstrated responses of each species to the cue combinations varying with the primary foraging mode of a predator. Predators involved in this study appear to respond more strongly to cues of the more locally abundant prey species, $P$. reticulata, particularly in the case of the top predator C. alta.

The importance of foraging mode in determining the relevance of prey cue type on predator behaviour in the present study may provide an explanation for earlier findings that damage-released chemical cues did not function as predator attractants. Specifically, Cashner [34] found that juvenile spotted bass Micropterus punctulatus did not demonstrate an attraction to the chemical cues of a suite of sympatric prey species. However, M. punctulatus may rely more on visual cues than chemical ones, as this species tends to be actively foraging, solitary predators. Although Chivers et al. [35] demonstrated attraction of the visually foraging, ambush predator northern pike Esox lucius to the chemical cues of fathead minnows Pimephales promelas; their experiment involved releasing chemical cues over a 30minute period. Similar experiments have been conducted over even longer timescales (hours, [19]). The present study involves five-minute observations, which may be a more ecologically relevant timeframe due to the mechanism of release and intransigence of damage-released chemical cues. Interspecific trophic differences may also be insufficient to predict responses to heterospecific chemical cues. In addition to intraspecific differences in predator behaviour and prey size preference [36], recent findings [2] have established a relative size threshold between antipredator and foraging responses to damage-released chemical cues (predator $L_{S}>$ $150 \%$ prey $L_{S} ;[17]$ ) as well as the ability of predators to determine prey quality and condition from information conveyed by these cues. Earlier studies (e.g., [34]) may have failed to include predators above such a relative size threshold or chemical cue donors of ideal size or condition and consequently were not able to elicit foraging responses in heterospecific receivers. 
As the top fish predator in this section of the Aripo River [25], C. alta preys upon smaller individuals of both of the other predatory species involved in this study and is likely to compete for forage opportunities with larger heterospecific size classes. Likely as a result of these predatory and trophic interactions, $A$. bimaculatus and $A$. pulcher are rarely observed in close proximity to $C$. alta (personal observations), whose presence may indicate relatively high levels of risk. The presence of significant linear relationships between predator species-pair counts in response to $P$. reticulata cues and insignificant relationships in response to $R$. hartii cues is consistent with the notion that predators generally display greater attraction responses to the cues of more abundant or familiar prey species. This observation may imply that $A$. bimaculatus and $A$. pulcher adopt riskier foraging strategies and enter potentially more dangerous areas when presented with more familiar foraging opportunities, increasing the likelihood of encountering C. alta and potentially incurring the risks of interspecific competition and/or predation. An adaptationist hypothesis for the evolution of this damagereleased chemical signalling system is that, in addition to the survival benefits accrued to conspecific chemical cue receivers through antipredator behavioural responses, chemical signalling may be advantageous to the sender by attracting secondary predators [15]. The differences in localized species-pair abundances in response to less familiar prey cues described above lend some support to this predator attraction/interference hypothesis, in that predators of lower trophic levels appear to avoid predators or competitors of higher trophic levels, sacrificing foraging opportunities in the process.

Prey fishes may experience increased mortality under conditions which eliminate sources of information on the level of predation risk (e.g., damage-released chemical cues lose functionality at $\mathrm{pH}<6.6$; [37]). The attraction of predators to heterospecific chemical cues demonstrated in the current study suggests that predators may be similarly deprived of information on the presence and quality of foraging opportunities under certain environmental conditions. Predators similarly deprived of sensory information may consequently experience negative fitness consequences. The differences in response in predator species and species-pairs to cues from different prey suggest directions for further research into both the fitness benefits accrued from the use of information on prey availability as well as the interactions between predator species in the context of predator interference.

\section{Acknowledgments}

The authors wish to thank the Director of Fisheries in the Trinidadian Ministry of Agriculture, Land and Marine Resources for permission to collect fish for use in this study. They also thank J.-G. J. Godin and I. W. Ramnarine for discussions on experimental design and potential locations for the present study and C. J. Macnaughton, P. H. Malka, and two anonymous reviewers for comments on an earlier version of this paper. Financial support was provided by Concordia University, le Fonds québécois de la Recherché sur la Nature et les Technologies (FQRNT) to C. K. Elvidge and the Natural Sciences and Engineering Research Council of Canada (NSERC) to G. E. Brown. All work reported herein was conducted in accordance with the guidelines of the Canadian Council on Animal Care and the laws of Canada and was approved by the Concordia University Animal Research Ethics Committee (Protocol no. AREC2008-BROW).

\section{References}

[1] E. Danchin, L. A. Giraldeau, T. J. Valone, and R. H. Wagner, "Public information: from nosy neighbors to cultural evolution," Science, vol. 305, no. 5683, pp. 487-491, 2004.

[2] C. K. Elvidge, I. W. Ramnarine, J. G. J. Godin, and G. E. Brown, "Size-mediated response to public cues of predation risk in a tropical stream fish," Journal of Fish Biology, vol. 77, no. 7, pp. 1632-1644, 2010.

[3] T. W. Cronin, "The visual ecology of predator-prey interactions," in Behavioural Ecology of Teleost Fishes, J. G. J. Godin, Ed., pp. 105-138, Oxford University Press, Oxford, UK, 1997.

[4] J. W. Kim, G. E. Brown, I. J. Dolinsek, N. N. Brodeur, A. O. H. C. Leduc, and J. W. A. Grant, "Combined effects of chemical and visual information in eliciting antipredator behaviour in juvenile Atlantic salmon Salmo salar," Journal of Fish Biology, vol. 74, no. 6, pp. 1280-1290, 2009.

[5] B. A. Hazlett and C. McLay, "Responses to predation risk: alternative strategies in the crab Heterozius rotundifrons," Animal Behaviour, vol. 69, no. 4, pp. 967-972, 2005.

[6] J. M. Hemmi, "Predator avoidance in fiddler crabs: 2. The visual cues," Animal Behaviour, vol. 69, no. 3, pp. 615-625, 2005.

[7] B. D. Wisenden, J. Pogatshnik, D. Gibson, L. Bonacci, A. Schumacher, and A. Willett, "Sound the alarm: learned association of predation risk with novel auditory stimuli by fathead minnows (Pimephales promelas) and glowlight tetras (Hemigrammus erythrozonus) after single simultaneous pairings with conspecific chemical alarm cues," Environmental Biology of Fishes, vol. 81, no. 2, pp. 141-147, 2008.

[8] M. C. O. Ferrari, B. D. Wisenden, and D. P. Chivers, "Chemical ecology of predator-prey interactions in aquatic ecosystems: a review and prospectus," Canadian Journal of Zoology, vol. 88, no. 7, pp. 698-724, 2010.

[9] R. J. F. Smith, "Avoiding and deterring predators," in Behavioural Ecology of Teleost Fishes, J. G. J. Godin, Ed., pp. 163-190, Oxford University Press, Oxford, UK, 1997.

[10] J. L. Golub, V. Vermette, and G. E. Brown, "Response to conspecific and heterospecific alarm cues by pumpkinseeds in simple and complex habitats: field verification of an ontogenetic shift," Journal of Fish Biology, vol. 66, no. 4, pp. 10731081, 2005.

[11] G. E. Brown and R. J. F. Smith, "Conspecific skin extracts elicit antipredator responses in juvenile rainbow trout (Oncorhynchus mykiss)," Canadian Journal of Zoology, vol. 75, no. 11, pp. 1916-1922, 1997.

[12] G. E. Brown, D. P. Chivers, and R. J. Smith, "Localized defecation by pike: a response to labelling by cyprinid alarm pheromone?" Behavioral Ecology and Sociobiology, vol. 36, no. 2, pp. 105-110, 1995.

[13] B. D. Wisenden, J. Karst, J. Miller, S. Miller, and L. Fuselier, "Anti-predator behaviour in response to conspecific chemical alarm cues in an esociform fish, Umbra limi (Kirtland 1840)," Environmental Biology of Fishes, vol. 82, no. 1, pp. 85-92, 2008. 
[14] G. E. Brown and J. G. J. Godin, "Chemical alarm signals in wild Trinidadian guppies (Poecilia reticulata)," Canadian Journal of Zoology, vol. 77, no. 4, pp. 562-570, 1999.

[15] R. J. F. Smith, "Alarm signals in fishes," Reviews in Fish Biology and Fisheries, vol. 2, no. 1, pp. 33-63, 1992.

[16] M. C. Harvey and G. E. Brown, "Dine or dash?: ontogenetic shift in the response of yellow perch to conspecific alarm cues," Environmental Biology of Fishes, vol. 70, no. 4, pp. 345-352, 2004.

[17] O. A. Popova, "The role of predaceous fish in ecosystems," in Ecology of Freshwater Fish Production, S. D. Gerking, Ed., pp. 215-249, Blackwell Scientific, Oxford, UK, 1978.

[18] G. E. Brown, V. J. Leblanc, and L. E. Porter, "Ontogenetic changes in the response of largemouth bass (Micropterus salmoides, Centrarchidae, Perciformes) to heterospecific alarm pheromones," Ethology, vol. 107, no. 5, pp. 401-414, 2001.

[19] B. D. Wisenden and T. A. Thiel, "Field verification of predator attraction to minnow alarm substance," Journal of Chemical Ecology, vol. 28, no. 2, pp. 433-438, 2002.

[20] S. L. Lima and L. M. Dill, "Behavioral decisions made under the risk of predation: a review and prospectus," Canadian Journal of Zoology, vol. 68, no. 4, pp. 619-640, 1990.

[21] A. Mathis, D. P. Chivers, and R. J. Smith, "Chemicl alarm signals: predator deterrents or predator attractants?" American Naturalist, vol. 145, no. 6, pp. 994-1005, 1995.

[22] O. M. Lonnstedt, M. I. McCormick, and D. P. Chivers, "Wellinformed foraging: damage-released chemical cues of injured prey signal quality and size to predators," Oecologia, vol. 168, no. 3, pp. 651-658, 2012.

[23] G. E. Brown, C. K. Elvidge, C. J. Macnaughton, I. Ramnarine, and J. G. J. Godin, "Cross-population responses to conspecific chemical alarm cues in wild Trinidadian guppies, Poecilia reticulata: evidence for local conservation of cue production," Canadian Journal of Zoology, vol. 88, no. 2, pp. 139-147, 2010.

[24] D. P. Croft, L. J. Morrell, A. S. Wade et al., "Predation risk as a driving force for sexual segregation: a cross-population comparison," American Naturalist, vol. 167, no. 6, pp. 867878, 2006.

[25] A. E. Magurran, Evolutionary Ecology: The Trinidadian Guppy, Oxford University Press, Oxford, UK, 2005.

[26] R. Froese and D. Pauly, "FishBase. 2011," World Wide Web electronic publication, (01/2010)http://www.fishbase.org/.

[27] J. Krause and J. G. J. Godin, "Predator preferences for attacking particular prey group sizes: consequences for predator hunting success and prey predation risk," Animal Behaviour, vol. 50, no. 2, pp. 465-473, 1995.

[28] K. E. Esteves, "Feeding ecology of three Astyanax species (Characidae, Tetragonopterinae) from a floodplain lake of Mogi-Guacu River, Parana River Basin, Brazil," Environmental Biology of Fishes, vol. 46, no. 1, pp. 83-101, 1996.

[29] B. H. Seghers, An analysis of geographic variation in the antipredator adaptations of the guppy, Poecilia reticulata [Ph.D. thesis], Department of Zoology, University of British Columbia, Vancouver BC, Canada, 1973.

[30] G. E. Brown, C. J. MacNaughton, C. K. Elvidge, I. Ramnarine, and J. G. J. Godin, "Provenance and threat-sensitive predator avoidance patterns in wild-caught Trinidadian guppies," Behavioral Ecology and Sociobiology, vol. 63, no. 5, pp. 699-706, 2009.

[31] J. Pinheiro, D. Bates, S. DebRoy, D. Sarkar, and R Development Core Team, "nlme: Linear and Nonlinear Mixed Effects Models," R package version 3.1-97, 2010.
[32] R Development Core Team, R: A Language and Environment for Statistical Computing, R Foundation for Statistical Computing, Vienna, Austria, 2010.

[33] E. Paradis, J. Claude, and K. Strimmer, "APE: analyses of phylogenetics and evolution in R language," Bioinformatics, vol. 20, no. 2, pp. 289-290, 2004.

[34] M. F. Cashner, "Are spotted bass (Micropterus punctulatus) attracted to Schreckstoff? A test of the predator attraction hypothesis," Copeia, no. 3, pp. 592-598, 2004.

[35] D. P. Chivers, G. E. Brown, and R. J. F. Smith, "The evolution of chemical alarm signals: attracting predators benefits alarm signal senders," American Naturalist, vol. 148, no. 4, pp. 649659, 1996.

[36] P. A. Nilsson and C. Bronmark, "Prey vulnerability to a gapesize limited predator: behavioural and morphological impacts on Northern pike piscivory," Oikos, vol. 88, no. 3, pp. 539-546, 2000.

[37] A. O. H. C. Leduc, J. M. Kelly, and G. E. Brown, "Detection of conspecific alarm cues by juvenile salmonids under neutral and weakly acidic conditions: laboratory and field tests," Oecologia, vol. 139, no. 2, pp. 318-324, 2004. 

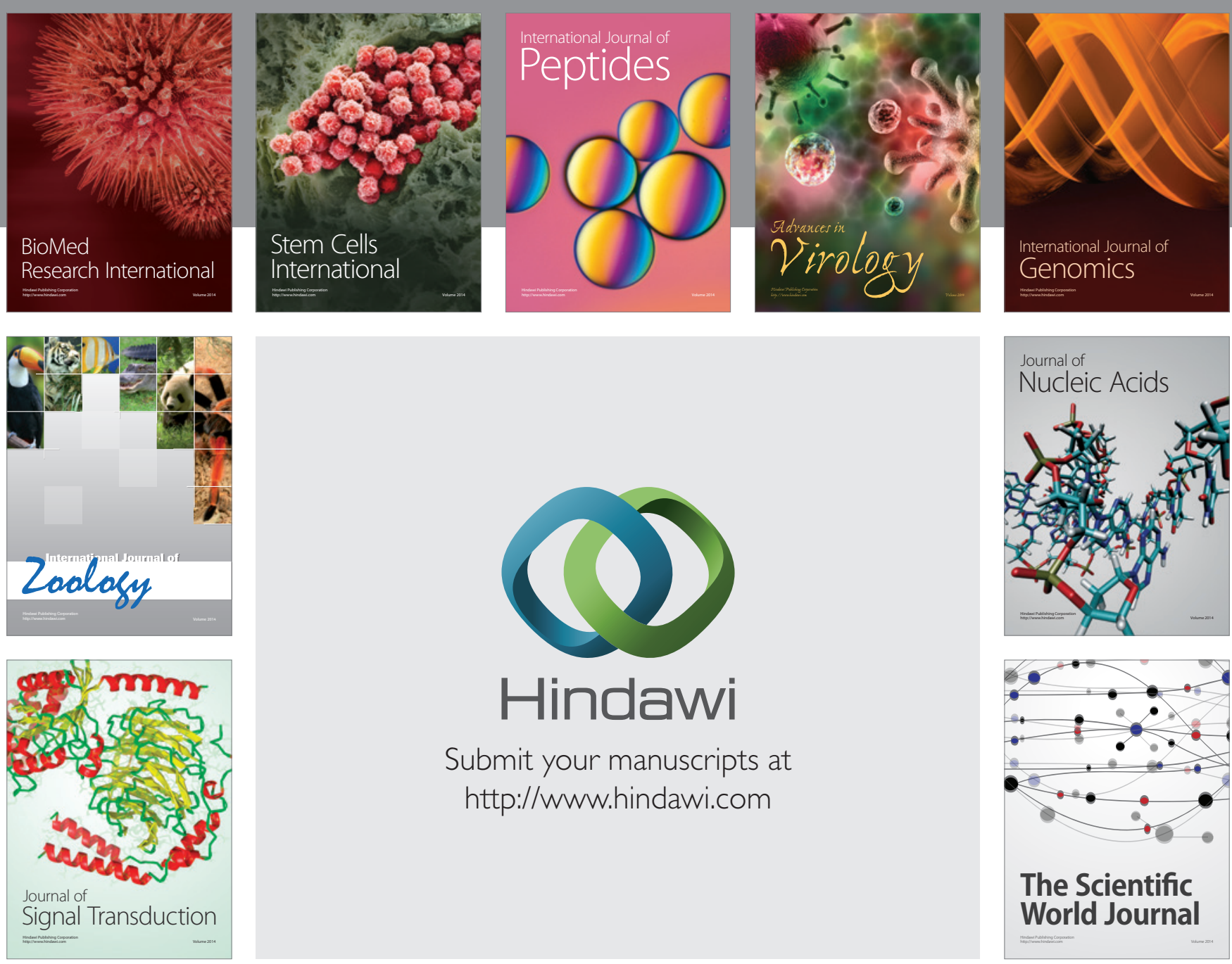

Submit your manuscripts at

http://www.hindawi.com
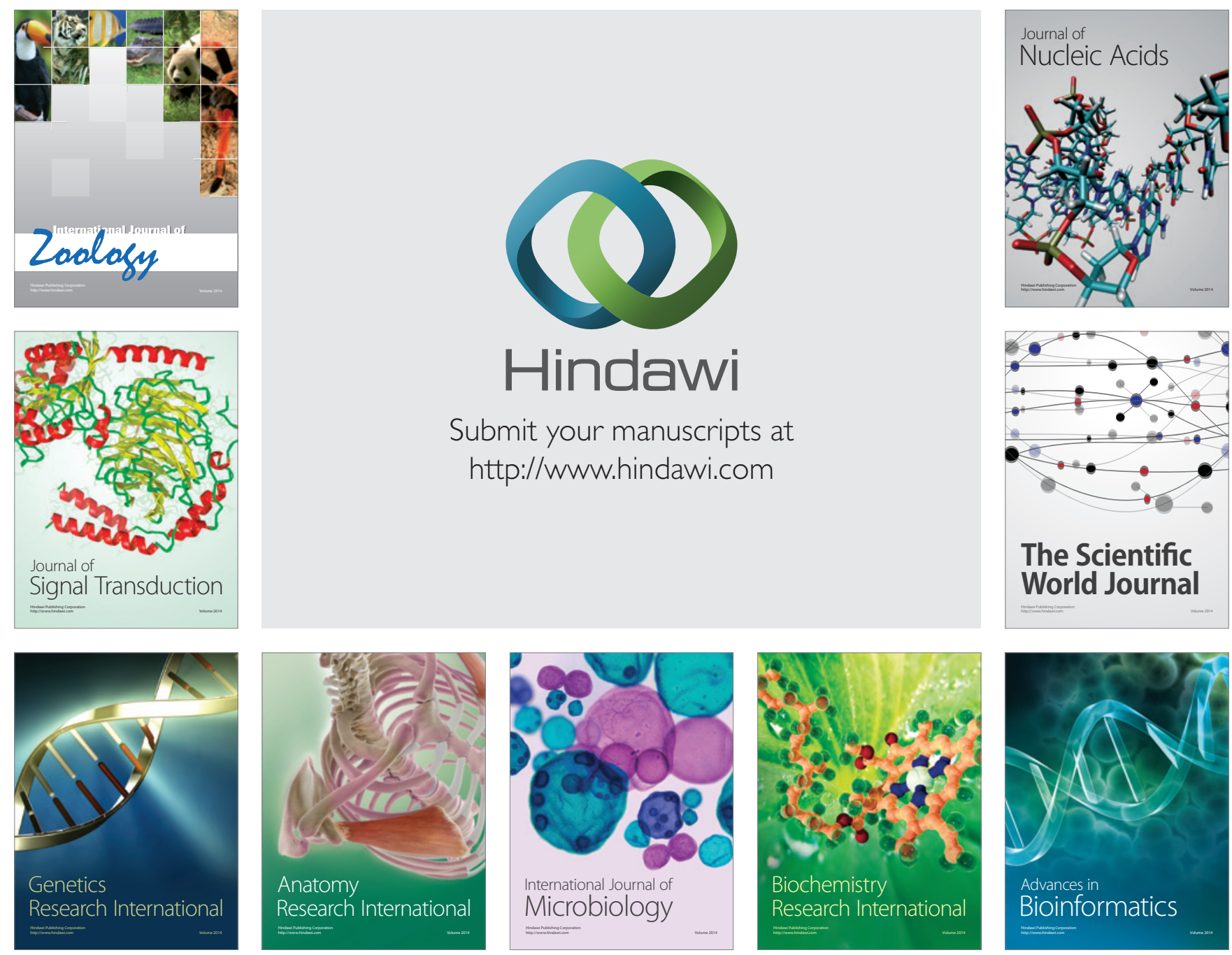

The Scientific World Journal
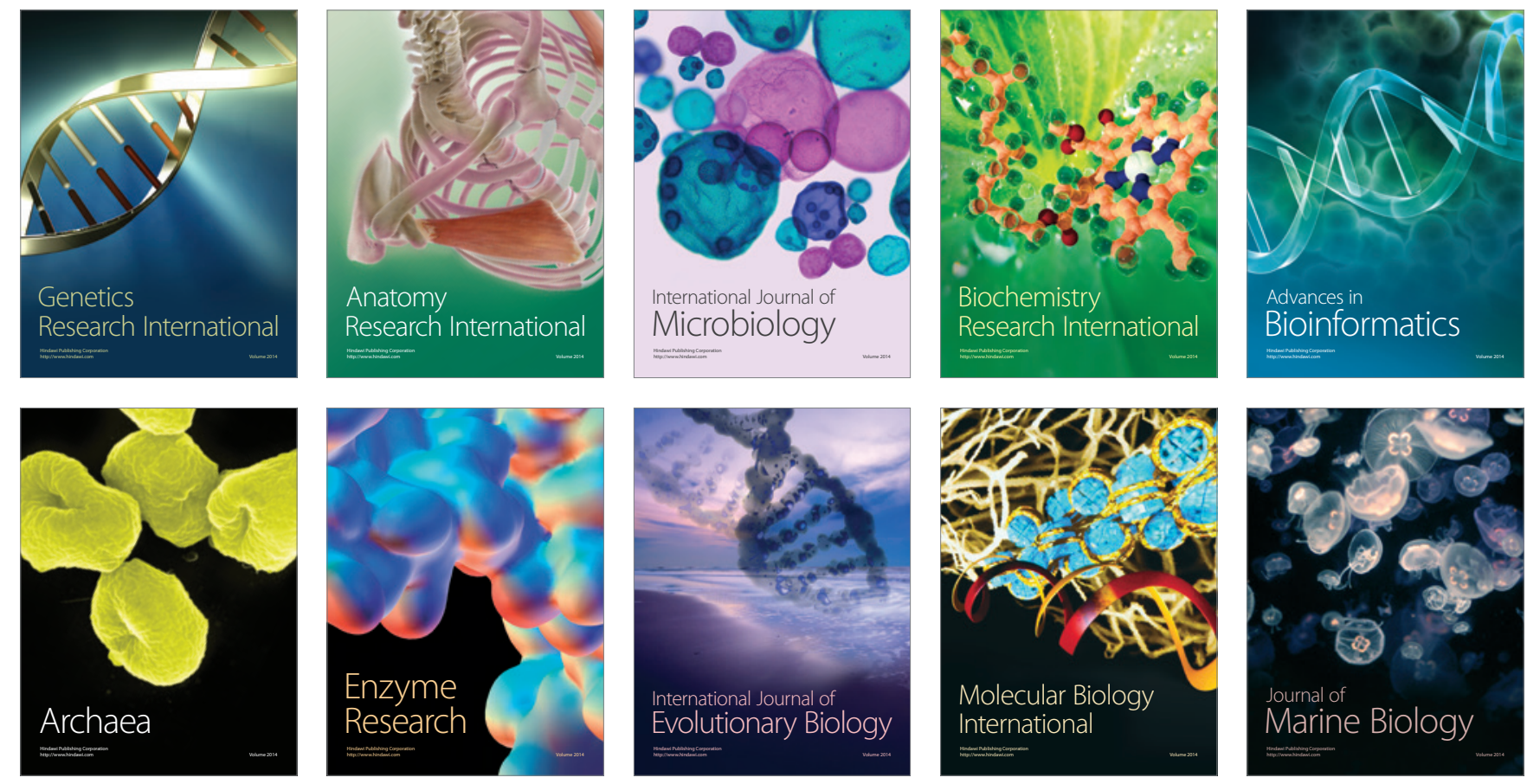